${ }^{1} \mathrm{~J}$. Geiss and H. Reeves, Astron. Astrophys. 18, 126 (1972).

${ }^{2}$ M. Meneguzzi, J. Audouze, and H. Reeves, Astron. Astrophys. 15, 337 (1971).

${ }^{3}$ W. A. Fowler, in Cortona Symposium on the Astrophysical Aspects of the Weak Interactions, edited by L. Radicati (Accademia Nazionale dei Lincei, Rome, Italy, 1971).

${ }^{4}$ R. V. Wagoner, W. A. Fowler, and F. Hoyle, Astrophys. J. 148, 3 (1967).

${ }^{5}$ H. Reeves, J. Audouze, W. A. Fowler, and D. Schramm, Astrophys. J. (to be published).

${ }^{6} \mathrm{~K}$. S. Thorne, Astrophys. J. 148, 51 (1967).

${ }^{7} J$. Silk and S. L. Shapiro, Astrophys. J. 166, 249 (1971).
${ }^{8}$ Y. B. Zel'dovich, Sov. J. At. Energy, 14, 83 (1964).

${ }^{9}$ J. W. Truran and A. G. W. Cameron, report, 1971 (unpublished).

${ }^{10}$ I. J. Danziger, Annu. Rev. Astron. Astrophys. 8, 161 (1970).

${ }^{11}$ R. R. Zappala, Lick Observatory Report No. 342, 1971 (unpublished).

${ }^{12} \mathrm{~J}$. G. Cohen, Ph.D. thesis, California Institute of Technology, 1971 (unpublished).

${ }^{13}$ R. V. Wagoner, Astrophys. J. Suppl. 18, 247 (1969).

${ }^{14}$ A. G. W. Cameron and W. A. Fowler, Astrophys. J. 164, 111 (1971).

${ }^{15}$ J. H. Oort, Astron. Astrophys. 7, 405 (1970).

PHYSICAL REVIEW D

\title{
Can Gravitation Have a Finite Range?
}

David G. Boulware*

Physics Department, University of Washington, Seattle, Washington 98195

and

\section{S. Deser $\dagger$}

Physics Department, Brandeis University, Waltham, Massachusetts 02154⿻ and Laboratoire de Physique Théorique et Hautes Energies, Orsay, France (Received 7 June 1972)

\begin{abstract}
No acceptable tensor gravitational theory with arbitrarily long but finite range exists. In linear approximation, the infinite-range limit is a scalar-tensor mixture implying an effective matter-matter coupling different from the strictly infinite-range prediction and contradicted by experiment. Compensation of the scalar requires the admixture of a ghost scalar coupling. In the massive version of the full Einstein theory, (a) there are necessarily six rather than the five tensor degrees of freedom, (b) the energy has no lower bound, (c) the infinite-range limit seems not to exist at all, and (d) lowest-order forces are the same as in the massive linearized theory.
\end{abstract}

\section{INTRODUCTION AND SUMMARY}

In the absence of experimental evidence to the contrary, it is simplest and most economical to ascribe the known long-range forces (electromagnetism and gravitation) to exchange of strictly massless quanta corresponding to an infiniterange interaction. One is then tempted to elevate the properties peculiar to massless vector and tensor fields, gauge and coordinate invariance together with the absence of lower helicity excitations, to the status of fundamental principles. There is, however, an equally basic principle, physical continuity, which demands that a theory be "stable" in its predictions, i.e., no more isolated from nearby models than our finite observations warrant. In particular, a good theory of long-range forces should have a smooth limit as the range tends to infinity, and this limit should agree with the strictly infinite-range model. This viewpoint has been forcefully stated for electrodynamics by Schrödinger, ${ }^{1}$ and it has been amply demonstrated by analysis of massive vector theory $^{2}$ that approximate gauge invariance is not the contradiction it first seems. There exists a continuum of consistent finite-mass photon theories whose consequences (both classical and quantum) smoothly approach those of conventional electrodynamics in the limit. Specifically, the longitudinal photons decouple from the current, becoming free fields (apart from gravitational interaction) while the helicity $\pm \mathbf{1}$ quanta become the transverse Maxwell photons in a guage-invariant way. The same continuity holds for nonrelativistic Newtonian gravitation, the geometric (but scalar) Nordström theory, and generally for exchange of 
spin-0 or $-\frac{1}{2}$ particles in special relativity. The only effective difference is in the exchange denominators (producing $e^{-m r} / r$ rather than $1 / r$ as the long-range potential); this difference disappears in the limit.

A conflict between the continuity and gauge principles first occurs for the non-Abelian Yang-Mills field, whose finite-mass counterpart seems consistent but does not tend to a complete transverselongitudinal decoupling at the quantum level. ${ }^{3}$ The problems only begin with closed loops, the limit being smooth for tree diagrams describing onequantum exchange. We shall be concerned here with the spin-2 theory, and the discrepancies it presents already at the classical level.

The free linear massive field does, essentially, ${ }^{4}$ smoothly approach in the limit a sum of massless spin-2, -1 , and -0 fields (from the parts of helicity 2,1 , and 0 ), the spin-2 part being identical to a strictly massless field. The latter is the linearized Einstein field with an Abelian gauge group. However, when sources are included, the situation is radically different from electrodynamics. As first shown by Van Dam and Veltman, ${ }^{3,5}$ the effective matter-matter interaction (through conserved stress tensors) is distinct from that predicted by the linearized Einstein field, however long the range. This violates the physical continuity argument: The predicted bending of light by the sun, for example, is $\frac{3}{4}$ that predicted by the zero-mass theory even if the gravitational range is larger than the Hubble radius, and is contradicted by experiment. ${ }^{6}$

The above discrepancy cannot be patched up by modifications of either the interaction or the free field. The basis of the effect is as follows: The helicity-2 modes and their coupling to the source correctly approach the $m=0$ theory in the limit. The helicity-1 modes decouple (as do the longitudinal photons in electrodynamics) and become a free Maxwell field, but the helicity -0 modes continue to interact (as a scalar field) with the trace of the source and yield an additional attractive interaction. Since any positive-energy scalar always leads to attraction, only a ghost (negativeenergy) scalar admixture can compensate the helicity -0 contribution.

The above results are not conclusive in themselves, since linear spin-2 theory is, at best, an approximate, not fully consistent, model of gravitation. In contrast to electrodynamics, where currents remain conserved when the coupling to the vector field (massive or massless) is taken into account, the stress tensor of any system cannot be conserved by virtue of its very coupling to the tensor field: A conserved source not including the spin-2 field is necessarily nondynamic, but a massless linear field can only consistently couple to a conserved source and the limit as $m \rightarrow 0$ can only exist if $\partial_{\mu} T^{\mu \nu} \rightarrow 0$. (Indeed, consistent coupling may be used ${ }^{7}$ as a basis for deriving the full nonlinear Einstein theory from the flat-space linear approximation.) We must, therefore, investigate the full Einstein theory coupled to dynamical systems and its behavior when the gauge (coordinate) invariance is broken by a finite-mass term. We will see that the difficulties become still worse than in the linear theory: Either the massless limit does not agree with general relativity or the energy has no lower bound, or both. Specifically, we will see that: (1) Any mass term leads to six rather than five degrees of freedom. (2) The energy has no lower bound, at least if the mass term agrees with its (ghost-free) linear form. [If it does not, then flat space (vacuum) is not a stable solution.] (3) The limit $m \rightarrow 0$ does not appear to exist at all. (4) For weak sources and excitations, the effective interaction due to onegraviton exchange is that of the linear finite-range model rather than the linearized approximation of general relativity $(m \equiv 0)$.

We must conclude that general relativity is an isolated theory and not the limit of a continuous set of long-range models. To be sure, there is at present no experimental evidence for a fallingoff of gravitation, and the isolation of the Einstein theory may, conversely, be taken as an argument in favor of its being the uniquely correct, purely geometrical theory of gravitation.

The outline of the paper is as follows:

We employ two approaches to the linear theory, taken to describe a spin-two (or in the case of zero mass, helicity-two) field (or particle) with positive-definite field energy. First, we consider the virtual exchange of spin-two particles, assuming that probabilities are all positive (positive Hilbert space norm). We find the results of Van Dam and Veltman ${ }^{3,5}$ described above completely independent of the form of the wave equation. Secondly, we describe the dynamics by an action and field equations and display in detail the zeromass limit, showing how the helicity \pm 2 modes of the finite-mass theory go over smoothly into the zero-mass helicity \pm 2 theory while the helicity \pm 1 modes decouple and the helicity -0 mode remains coupled, providing a scalar interaction which produces the experimental discrepancy.

The nonlinear theory is much more complicated and is not uniquely defined. We introduce the mass by adding (nonderivative) terms to the Einstein action. We then show that the mass term cannot be an invariant function of the metric but must involve some background metric which we take to be the Minkowski metric. The theory is 
then shown to have six rather than five degrees of freedom for any mass term. We then treat in more detail various mass terms and show that none provides a satisfactory theory - the energy is unbounded from below and there does not appear to exist a zero mass limit at all. We then show that, for any mass term such that flat space is a local stable equilibrium state, the lowest order mass term must be the same as that of the linear theory; hence, the experimental difficulties of the linearized theory for weak sources and excitations persist in the full theory. Finally, we discuss theories relating the $f$ meson to gravitation, arguing that the same difficulties are likely to appear there.

\section{THE LINEAR THEORY}

In this section, we analyze the dynamics of a linear massive spin-two system coupled to a conserved, prescribed tensor source $T^{\mu \nu}$. There are two approaches to the problem; we shall use both. First, we discuss the effects of the virtual exchange of spin-two quanta both of zero mass and of very small mass; and, secondly, we discuss the wave equation and Hamiltonian for tensor fields. The results are the same in the two analyses.

The amplitude for the emission of no gravitons by an external source $T^{\mu \nu}$ is $^{8}$

$$
\langle\text { out }| \text { in }\rangle=\exp \left(i \frac{1}{2} K_{m}^{2} \int d^{4} x d^{4} x^{\prime} T^{\mu \nu}(x) D_{\mu \nu, \lambda \sigma}^{m}\left(x-x^{\prime}\right) T^{\lambda \sigma}\left(x^{\prime}\right)\right),
$$

where $K_{m}$ is the coupling constant which we determine below and $D_{\mu \nu, \lambda \sigma}^{m}$ is the graviton propagator whose properties we must now discuss.

It is most convenient to treat the propagator in momentum space; there, it has a pole at ${ }^{9} p^{2}=-m^{2}$ and the residue of the pole must yield an appropriate spin projection operator. Thus,

$$
\tilde{D}_{\mu \nu, \lambda \sigma}^{m}(p) \equiv \int d^{4} x e^{-i p x} D_{\mu \nu, \lambda \sigma}^{m}(x)=\left(p^{2}+m^{2}-i \epsilon\right)^{-1} d_{\mu \nu, \lambda \sigma}^{m}(p) .
$$

For pure spin-two exchange, the residue at the pole must be the projection operator for spin two; $p^{\mu}$ is a timelike vector, hence a frame in which $p^{\mu}=\delta_{0}^{\mu} m$ may be chosen. In that frame $d_{\mu \nu, \lambda \sigma}^{m}$ can only have space components and the spin-two projection operator is

$$
d_{m n, l s}^{(2)}=\frac{1}{2}\left(\delta_{m l} \delta_{n s}+\delta_{m s} \delta_{n l}-\frac{2}{3} \delta_{m n} \delta_{l s}\right)
$$

which becomes

$$
d_{\mu \nu, \lambda \sigma}^{(2)}(p)=\frac{1}{2}\left(\theta_{\mu \lambda} \theta_{\nu \sigma}+\theta_{\mu \sigma} \theta_{\nu \lambda}-\frac{2}{3} \theta_{\mu \nu} \theta_{\lambda \sigma}\right),
$$

where $\theta_{\mu \nu} \equiv \eta_{\mu \nu}+\left(p_{\mu} p_{\nu} / m^{2}\right)$, upon transforming to an arbitrary frame.

The spin-one exchange involves the spin-one projection operator,

$$
d_{\mu \nu, \lambda \sigma}^{(1)}=p_{\mu} p_{\lambda} \theta_{\nu \sigma}+p_{\nu} p_{\lambda} \theta_{\mu \sigma}+p_{\mu} p_{\sigma} \theta_{\nu \lambda}+p_{\nu} p_{\sigma} \theta_{\mu \lambda}
$$

The factors of $p$ ensure that only $\partial_{\nu} T^{\mu \nu}$ contributes to the spin-one exchange (that is the only vector available). In the limit as $m \rightarrow 0$, the stress tensor must be conserved ${ }^{10}$; if it is taken to be conserved before the limit, spin-one exchange cannot contribute. ${ }^{11}$

Similarly, there can be no coupling through $\partial_{\mu} \partial_{\nu} T^{\mu \nu}$; the only scalar exchange is through $T_{\mu}^{\mu}$. Thus, the most general form for $d_{\mu \nu, \lambda \sigma}^{m}$ is, dropping all $p$ 's since the stres tensor is conserved,

$$
d_{\mu \nu, \lambda \sigma}^{m}=d_{\mu \nu, \lambda \sigma}^{(2)}+|\beta|^{2} \eta_{\mu \nu} \eta_{\lambda \sigma} \rightarrow \frac{1}{2}\left(\eta_{\mu \lambda} \eta_{\nu \sigma}+\eta_{\mu \sigma} \eta_{\nu \lambda}-\frac{2}{3} \eta_{\mu \nu} \eta_{\lambda \sigma}\right)+|\beta|^{2} \eta_{\mu \nu} \eta_{\lambda \sigma}
$$

The denominator, $p^{2}+m^{2}-i \epsilon$, was chosen so that only positive-energy gravitons are emitted; then, the over-all sign of $d$ and the appearance of $|\beta|^{2}$ are dictated by the requirement that the imaginary part of $D$ be positive, i.e., that gravitons be emitted with positive probability and that the probability of no emission be less than one, not greater. Note that the coefficient of $\eta_{\mu \nu} \eta_{\lambda \sigma}$ is greater than or equal to $-\frac{1}{3}$; no nonghost scalar admixture can bring the coefficient below $-\frac{1}{3} .{ }^{11}$ The properties of ghost admixtures are outlined in Appendix C.

So far we have based our considerations upon the real graviton emission; we now consider the effect on the amplitude for static $T^{\mu \nu}$. In this case, rather than having $p^{2}=-m^{2}$, we have $p^{0}=0$; and performing the $\overrightarrow{\mathrm{p}}$ integrals, we obtain 


$$
\begin{aligned}
\frac{1}{2} K_{m}{ }^{2} \int d^{4} x d^{4} x^{\prime} T^{\mu \nu}(x) D_{\mu \nu, \lambda \sigma}^{m}\left(x-x^{\prime}\right) T^{\lambda \sigma} & \left(x^{\prime}\right) \\
& =\frac{1}{2} K_{m}{ }^{2} \int d t \int d^{3} r d^{3} \gamma^{\prime} T^{\mu \nu}(\overrightarrow{\mathrm{r}})\left(\eta_{\mu \lambda} \eta_{\nu \sigma}-\frac{1}{3} \eta_{\mu \nu} \eta_{\lambda \sigma}+|\beta|^{2} \eta_{\mu \nu} \eta_{\lambda \sigma}\right) T^{\lambda \sigma}\left(\overrightarrow{\mathrm{r}}^{\prime}\right) \frac{e^{-m \mid \overrightarrow{\mathrm{r}}-\overrightarrow{\mathrm{r}}^{\prime \prime}}}{4 \pi\left|\overrightarrow{\mathrm{r}}-\overrightarrow{\mathrm{r}}^{\prime}\right|} \\
& \equiv-\int d t V .
\end{aligned}
$$

Upon comparison with Eq. (1) we see that $V$ may be identified as the potential energy. The energy of interaction between two well localized masses $M_{1}$ and $M_{2}$ separated by a distance $r$ is then

$$
-\frac{K_{m}^{2}}{4 \pi} \frac{M_{1} M_{2}}{r} e^{-m r}\left(\frac{2}{3}+|\beta|^{2}\right),
$$

and in order for it to yield the correct Newtonian force,

$$
K_{m}^{2}=\frac{4 \pi G}{\frac{2}{3}+|\beta|^{2}} .
$$

If we now consider the interaction of a mass with a light beam, for which $T_{\mu}^{\mu}=0$, we obtain

$$
-\frac{K_{m}^{2}}{4 \pi} \frac{M\left(E / c^{2}\right)}{r} e^{-m r}=-\frac{G M}{\frac{2}{3}+|\beta|^{2}} \frac{\left(E / c^{2}\right)}{r} e^{-m r},
$$

where $E$ is the energy carried by the light.

We now turn to the zero-mass exchange; the stress tensor now must be conserved, ${ }^{10}$ hence only the forms $\eta_{\mu \lambda} \eta_{\nu \sigma}$ and $\eta_{\mu \nu} \eta_{\lambda \sigma}$ need be considered. The most general form for $d_{\mu \nu, \lambda \sigma}^{0}$ is then

$$
d_{\mu \nu, \lambda \sigma}^{0}=\frac{1}{2}\left(\eta_{\mu \lambda} \eta_{\nu \sigma}+\eta_{\mu \sigma} \eta_{\nu \lambda}+a \eta_{\mu \nu} \eta_{\lambda \sigma}\right) \text {, }
$$

and we must impose the condition of pure helicity \pm 2 exchange. Note that here we impose helicity two rather than spin-two exchange. This is possible since the graviton has zero mass and it corresponds to the linearization of the Einstein theory. At the pole $p^{2}=0, T^{0 \lambda}(p)=T^{3 \lambda}(p)$ [if we take $p^{\mu}$ $\left.=p\left(\delta_{0}^{\mu}+\delta_{3}^{\mu}\right)\right]$ and only $T^{11}, T^{22}$, and $T^{12}=T^{21}$ can contribute; the helicity \pm 2 parts are $T^{11}-T^{22}$ and $T^{12}$, the helicity-zero part is proportional to $\left(\delta_{1}^{k} \delta_{1}^{e}+\delta_{2}^{k} \delta_{2}^{e}\right)$. Then, the helicity-zero part of $T$ will not contribute only if $d_{\mu \nu, \lambda \sigma}^{0}$ is traceless in the two-dimensional subspace orthogonal to $\delta_{0}^{\mu}$ and $\delta_{3}^{\mu}$, i.e., if $a=-1$. Then,

$$
d_{\mu \nu, \lambda \sigma}^{0}=\frac{1}{2}\left(\eta_{\mu \lambda} \eta_{\nu \sigma}+\eta_{\mu \sigma} \eta_{\nu \lambda}-\eta_{\mu \nu} \eta_{\lambda \sigma}\right)
$$

The energy of interaction between well localized masses is then

$$
\begin{aligned}
V & =-\frac{K_{0}{ }^{2}}{4 \pi} \frac{1}{2} \frac{M_{1} M_{2}}{r} \\
& =-G \frac{M_{1} M_{1}}{r} ;
\end{aligned}
$$

hence $K_{0}{ }^{2}=2(4 \pi G)$, and the interaction between a mass and light is

$$
V=-\frac{K_{0}^{2}}{4 \pi} \frac{M\left(E / c^{2}\right)}{r} .
$$

Thus, the finite-mass graviton exchange will produce a bending of light which is, for $\beta=0$, as was first pointed out by Van Dam and Veltman, ${ }^{3,5}$ $\frac{3}{4}$ that of the pure helicity-two zero-mass theory. From the general expression $\frac{3}{4}\left(1+\frac{3}{2}|\beta|^{2}\right)^{-1}$ we see that scalar exchange $(\beta \neq 0)$ only increases the discrepancy. Experiment yields a value within $10 \%$ of that predicted by the pure helicity \pm 2 , zeromass potential, Eq. (11), ruling out any finitemass, nonghost linearized theory. ${ }^{6}$ Also, the time delay, "fourth test" predictions will be affected by the same factor, although the red shift as measured by a massive clock will be unaffected, since it depends only on the Newtonian potential.

We now analyze the problem from the point of view of a classical field equation; in order for the system to be pure spin two, it is essential to use a particular, Pauli-Fierz, mass term. ${ }^{12}$ Otherwise, there will be admixtures of lower spin, in general with negative-energy ${ }^{13}$ (ghost) properties. The usual second-order form of the action governing the symmetric tensor field is

$$
\begin{aligned}
I=-\frac{1}{4} \int d^{4} x & \left\{h_{\mu \nu, \alpha} h^{\mu \nu, \alpha}-2 h_{\mu, \nu}{ }_{, \nu} h^{\mu \lambda}, \lambda\right. \\
& +2 h^{\mu \nu}{ }_{, \nu} h_{\alpha, \mu}^{\alpha}-h_{\alpha, \mu}^{\alpha} h_{\beta}^{\beta, \mu} \\
& \left.+m^{2}\left[h_{\mu \nu} h^{\mu \nu}-\left(h_{\alpha}^{\alpha}\right)^{2}\right]-2 \kappa_{m} h_{\mu \nu} T^{\mu \nu}\right\}
\end{aligned}
$$

and the field equations are

$$
\begin{aligned}
& -\partial^{2} h^{\mu \nu}+\partial^{\mu} h^{\nu \lambda}{ }_{, \lambda}+\partial^{\nu} h^{\mu \lambda}, \lambda-\eta^{\mu \nu} h^{\lambda \sigma}{ }_{, \lambda \sigma} \\
& -\left(\partial^{\mu} \partial^{\nu}-\eta^{\mu \nu} \partial^{2}\right) h^{\alpha}{ }_{\alpha}+m^{2}\left[h^{\mu \nu}-\eta^{\mu \nu} h_{\alpha}^{\alpha}\right]=K_{m} T^{\mu \nu} .
\end{aligned}
$$

We could have chosen any $\bar{h}_{\mu \nu}=h_{\mu \nu}+a \eta_{\mu \nu} h_{\lambda}^{\lambda}\left(a \neq-\frac{1}{4}\right)$ as the basic variable, which would yield a different form but identical results. This choice corresponds to a linearization of the Einstein field about the Minkowski metric $\eta_{\mu \nu}$ in terms of $h_{\mu \nu}$ $\equiv g_{\mu \nu}-\eta_{\mu \nu}$, rather than, say, $\sqrt{-g} g^{\mu \nu}-\eta^{\mu \nu}$.

Using the divergences and trace of the field equations leads to the five constraints 


$$
h^{\mu \nu}{ }_{, \nu}=\partial^{\mu} h_{\alpha}^{\alpha}, \quad h^{\alpha}{ }_{\alpha}=\left(1 / 3 m^{2}\right) T_{\alpha}^{\alpha},
$$

(as against the single source-independent $\partial_{\mu} A^{\mu}=0$ constraint in the vector case). Despite their appearance, these constraints do not correspond to gauge conditions in the limiting theory. This is particularly clear here, since (14) implies that the linear curvature scalar $R^{L}$ vanishes:

$$
R^{L} \equiv 2 \partial_{\mu}\left(h^{\mu \nu}{ }_{\nu}-\partial^{\mu} h_{\alpha}{ }^{\alpha}\right)
$$

whereas in the massless theory, the trace of (13) states that $R^{L}=T^{\alpha}{ }_{\alpha}$. But $R^{L}$ is invariant under the gauge group $\delta h_{\mu \nu}=\xi_{\mu, \nu}+\xi_{\nu, \mu}$ and so cannot be made to vanish by a gauge choice. Nor can (a fortiori) the combination $\left(h^{\mu \nu}{ }_{, \nu}-\partial^{\mu} h^{\alpha}{ }_{\alpha}\right)$ be set to zero in any gauge, unless $R^{L}$ vanishes, since the gauge functions would have to satisfy

$$
\begin{aligned}
0 & =\bar{h}^{\mu \nu}{ }_{, \nu}-\partial^{\mu} \bar{h}^{\alpha}{ }_{\alpha} \\
& =\left(h^{\mu \nu}{ }_{\nu}-\partial^{\mu} h^{\alpha}{ }_{\alpha}\right)+\left(\partial^{2} \eta^{\mu \nu}-\partial^{\mu} \partial^{\nu}\right) \xi_{\nu}
\end{aligned}
$$

which are consistent only if the "current" $\left(h^{\mu \nu}{ }_{\nu}-\partial^{\mu} h^{\alpha}{ }_{\alpha}\right)$ is conserved. These facts, along with the $1 / \mathrm{m}^{2}$ behavior of the constrained variables, imply that the limit $m \rightarrow 0$ will not be an obvious one.

The effective matter-matter interaction implied by Eq. (13) is easily obtained by using Eq. (14) to write ${ }^{14}$

$$
\begin{aligned}
\left(-\partial^{2}+m^{2}\right) h^{\mu \nu} & =K_{m}\left(T^{\mu \nu}-\frac{1}{3} \eta^{\mu \nu} T_{\alpha}^{\alpha}+\frac{1}{3} m^{-2} \partial^{\mu} \partial^{\nu} T_{\alpha}^{\alpha}\right) \\
& \equiv S^{\mu \nu} .
\end{aligned}
$$

This in turn yields the effective one-graviton-exchange interaction

$$
\begin{aligned}
I(m) & =\frac{1}{2} K_{m} \int d^{4} x d^{4} x^{\prime} T^{\mu \nu}(x) \Delta\left(x-x^{\prime}, m^{2}\right) S_{\mu \nu}\left(x^{\prime}\right) \\
& =\frac{1}{2} K_{m}^{2} \int d^{4} x d^{4} x^{\prime} T^{\mu \nu}(x) D_{\mu \nu, \lambda \sigma}^{m}\left(x-x^{\prime}\right) T^{\lambda \sigma}\left(x^{\prime}\right) .
\end{aligned}
$$

Completely analogous arguments lead to the wellknown form

$$
\begin{gathered}
I(m=0)=\frac{1}{2} K_{0}^{2} \int d^{4} x d^{4} x^{\prime} T^{\mu \nu}(x) \Delta\left(x-x^{\prime}, 0\right) \\
\quad \times\left[T_{\mu \nu}\left(x^{\prime}\right)-\frac{1}{2} \eta_{\mu \nu} T_{\alpha}^{\alpha}\left(x^{\prime}\right)\right] \\
=\frac{1}{2} K_{0}^{2} \int d^{4} x d^{4} x^{\prime} T^{\mu \nu}(x) D_{\mu \nu, \lambda \sigma}^{o}\left(x-x^{\prime}\right) T^{\lambda \sigma}\left(x^{\prime}\right) .
\end{gathered}
$$

We have found the same forms as before with, of course, the same experimental consequences.

Strictly speaking, the motion of a body in a gravitational field goes beyond the linear approxi- mation (in which $T^{\mu \nu}{ }_{, \nu}=0$ ) since the geodesic equation is really a consequence of covariant conservation $T_{; \nu}^{\mu \nu}=0$ in an external metric. However, the effective matter-matter interaction is equivalent to the geodesic equation (to first order in the field) if we add it (somewhat inconsistently) to the free action of a particle and consider $T_{\mu \nu}$ to be the sum of a part referring to a fixed source of $h_{\mu \nu}$ and the free $t_{\mu \nu}$ of the particle. In this framework, the particle's motion will be governed by the "Schwarzschild metric" of the source. We can compute the latter in the usual way as the exterior time-independent spherically symmetric solution of (13) [or, equivalently, by setting $T^{00}$ $=M \delta^{(3)}(\overrightarrow{\mathrm{r}}) T^{0 i}=0=T^{i j}$ there]. Spherical symmetry implies that

$$
h_{00}=\eta(r), \quad h_{0 i}=\nabla_{i} p(r),
$$

and

$$
h_{i j}=\left(\delta_{i j} \nabla^{2}-\nabla_{i} \nabla_{j}\right) f(r)+\delta_{i j} k(r) .
$$

The conditions $h_{i i}=h_{00}$ and $h_{i j, j}=0=h_{0 i, i}$ imply

$$
3 k+2 \nabla^{2} f=\eta, \quad k=0, \quad \text { and } \nabla^{2} p=0 .
$$

The field equations then yield

$$
\eta=\alpha Y \text { and } p=0 \text {, }
$$

where $Y \equiv\left(e^{-m r} / r\right)$. Normalizing $\alpha$ to its Newtonian value $2 G M$ the field has the form

$$
\begin{aligned}
h_{00} & =2 G M Y(r) \underset{m \rightarrow 0}{\sim}(2 M G / r), \quad h_{0 i}=0, \\
h_{i j} & =G M \delta_{i j} Y-G M m^{-2} \nabla_{i} \nabla_{j} Y \\
& \underset{m \rightarrow 0}{\sim}(G M / r) \delta_{i j}-G M m^{-2} \nabla_{i} \nabla_{j}\left(e^{-m r} / r\right) .
\end{aligned}
$$

This is to be compared to the linearized Einstein metric. The latter may be written in arbitrary static spherically symmetric gauge $\xi_{\mu}=\left(\xi_{0}, \nabla_{i} \xi\right)$ starting from its isotropic form, as

$$
\begin{aligned}
& h_{00}=(2 G M / r), \quad h_{0 i}=\nabla_{i} \xi_{0}, \\
& h_{i j}=(2 G M / r) \delta_{i j}-\nabla_{i} \nabla_{j} \xi .
\end{aligned}
$$

(The usual Schwarzschild coordinates are reached by $\xi_{0}=0, \xi=2 G M r$.) Comparing (22) with (23), we see that with the Newtonian limits normalized (equal $h_{00}$ ), the "massive" spatial metric has a singular $O\left(\mathrm{~m}^{-2}\right)$ gauge part and a remainder which is $\frac{1}{2}$ the Einstein value. Since photons feel equal contributions from $h_{00}$ and $h_{i j}$, this is the source of the $\frac{3}{4}$ factor in bending. Note that the trace of the gauge part is finite, so that $h_{i i}(m) \rightarrow 2 G M / r$ $=h_{00}^{(m)}$, as required, while the $h^{\alpha}{ }_{\alpha}=0$ condition only holds in Schwarzschild coordinates for the massless metric. The latter cannot simultaneously satisfy the five gauge conditions $h^{\mu \nu}{ }_{, \nu}=0=h^{\alpha}{ }_{\alpha}$. Therefore, any motion to which $h_{i j}$ contributes 
( $v^{2} / c^{2}$ corrections) will be different here. One could also discuss perihelion precession and obtain a radical deviation from the usual value, but such a calculation ceases to be meaningful in the linearized approximation, depending too strongly on further detailed assumptions.

We now turn to the analysis of the action rather than the field equations. This will exhibit the detailed behavior of the degrees of freedom of the field in the limit and serves as a preface to the formally analogous full Einstein theory.

The action of the full Einstein theory may be put in the canonical form ${ }^{15}$

$$
\begin{aligned}
I & =\int{ }^{4} R d^{4} x \\
& =\int d^{4} x\left(\pi^{i j} \dot{g}_{i j}-N R^{0}-N_{i} R^{i}\right),
\end{aligned}
$$

where $N \equiv\left(-g^{00}\right)^{-1 / 2}, N_{i} \equiv g_{0 i}$, and the $R^{\mu}$ are functions of the $g_{i j}$ and their conjugate momenta $\pi^{i j}$, but not of the $\left(N, N_{i}\right)$. Specifically,

$$
-R^{0} \equiv 3 \mathbb{R}+g^{-1 / 2}\left(\frac{1}{2} \pi^{2}-\pi^{i j} \pi_{i j}\right)
$$

and

$$
R^{i} \equiv-2 \pi^{i j}{ }_{1 j}
$$

where ${ }^{3} R, \sqrt{g}$ and the covariant (stroke) derivative are all with respect to the spatial metric $g_{i j}$. We may linearize about the Minkowski metric by expanding to quadratic order in $h_{\mu \nu}=g_{\mu \nu}-\eta_{\mu \nu}$; in particular, $N \sim 1-\frac{1}{2} h_{00}+O\left(h^{2}\right)$. We obtain for the linearized theory's action,

$$
I_{L}(m=0)=\int d^{4} x\left[\pi^{i j} \dot{g}_{i j}-\left(\pi^{i j} \pi_{i j}-\frac{1}{2} \pi^{2}\right)+2 N_{i} \pi_{, j}^{i j}+\frac{1}{2} h_{00}\left(\nabla^{2} h_{i i}-h_{i j, i j}\right)+{ }^{3} \mathbb{R}^{Q}+\frac{1}{2} h_{i j} T^{i j}+N_{i} T^{0 i}+\frac{1}{2} h_{00} T^{00}\right]
$$

where the coupling $\frac{1}{2} h_{\mu \nu} T^{\mu \nu}$ has been included and ${ }^{3} \mathscr{R}^{Q}$ is the three-dimensional scalar curvature density to quadratic order in $h_{i j}$ :

$$
\int d^{3} r^{3} \mathcal{R}^{Q}=-\frac{1}{4} \int d^{3} r\left[\left(h_{i j, k}\right)^{2}-2\left(h_{i}\right)^{2}+2 h_{i} h_{, i}-\left(h_{, i}\right)^{2}\right]
$$

with the abbreviations $h_{i} \equiv h_{i j, j}, h \equiv h_{i i}$ for the spatial components. The action (26) is of course the same as (12) for $m \rightarrow 0$, but in first-order form. The mass term, in the same notation, is

$$
\begin{aligned}
I_{m} & =-m^{2 \frac{1}{4}} \int d^{4} x\left[h_{\mu \nu} h^{\mu \nu}-\left(h^{\alpha}{ }^{2}\right)^{2}\right. \\
& =-\frac{1}{4} m^{2} \int d^{4} x\left(h_{i j}{ }^{2}-h^{2}+2 h h_{00}-2{N_{i}}^{2}\right) .
\end{aligned}
$$

We see immediately, in both the massless theory and the massive theory, that $h_{00}$ is a Lagrange multiplier whose variation yields one constraint,

$$
\nabla^{2} h-h_{i j, i j}+T^{00}=m^{2} h
$$

thereby eliminating one of the six a priori degrees of freedom (as well as $h_{00}$ itself) just as the form $A_{0}(\vec{\nabla} \cdot \overrightarrow{\mathrm{E}}-\rho)$ eliminates the longitudinal photons in electrodynamics upon varying $A_{0}$. The $N_{i}$ were also Lagrange multipliers in the massless theory eliminating three further degrees of freedom and leaving the two helicity \pm 2 modes as the dynamical variables. However, because the mass term is quadratic in $N_{i}$, the constraint now determines $N_{i}$ itself,

$$
N_{i}=m^{-2}\left(2 \pi^{i j}{ }_{j}+T^{0 i}\right)
$$

rather than eliminating other variables. This leaves the five degrees of freedom of the massive theory just as the condition $A^{0}=m^{-2}\left(j^{0}-\vec{\nabla} \cdot \overrightarrow{\mathrm{E}}\right)$ in massive electrodynamics replaces the Gauss equation, per mitting excitation of longitudinal photons. Note also that any other mass term ${ }^{16}\left[h_{\mu \nu}{ }^{2}-a\left(h^{\alpha}{ }_{\alpha}\right)^{2}\right](a \neq 1)$ would necessarily be quadratic in $h_{00}$ also and so correspond to six degrees of freedom. (It corresponds to a general tensor-scalar mixture at finite $m$.)

To disentangle the contributions of the various helicity components, we perform a decomposition of the variables $h_{i j}$ and $\pi^{i j}$ into orthogonal components according to

$$
h_{i j}=h_{i j}^{T T}+h_{i, j}^{T}+h_{j, i}^{T}+\frac{1}{2}\left(\delta_{i j}-\nabla_{i} \nabla_{j} \nabla^{-2}\right) h^{T}+2 h_{, i j}^{L}
$$

with the trace and divergence properties

$$
h_{i j, j}^{T T} \equiv 0 \equiv h_{i i}^{T T}, \quad h_{i, i}^{T} \equiv 0, \quad h_{i i}=h^{T}+2 \nabla^{2} h^{L}
$$


and, for any two tensors, the orthogonality relation

$$
\int V_{i j}^{a} W_{i j}^{b} d^{3} r=0, \quad a \neq b
$$

holds among different components of (30a). The transverse-traceless tensors and transverse vectors represent helicity 2 and 1 , respectively, while $\left(h^{T}, h^{L}\right)$ are mixtures of zero-helicity and zero-trace (scalar) pieces. We may now eliminate the constraints in terms of the appropriate orthogonal components. In addition to eliminating $N_{i}$ by $(29 \mathrm{~b})$ and $h^{L}$ by $(29 \mathrm{a})$, written as

$$
\nabla^{2} h_{L}=\left(2 m^{2}\right)^{-1}\left[-\left(-\nabla^{3}+m^{2}\right) h^{T}+T^{00}\right]
$$

we use the relation implicit in I that $\pi_{i i}=N_{i, i}$ (corresponding to $h_{0, \lambda}^{\lambda}-h_{\alpha, 0}^{\alpha}=0$ ), to eliminate $\pi^{T}$,

$$
\pi^{T}=-2 \pi^{l}, l+N_{l, l} \text {. }
$$

Inserting the orthogonal decompositions $(30 \mathrm{a})$, and the relations $(29 \mathrm{~b}, 31,32)$ into the total action, we have, after straightforward manipulations, the following form for $I_{L}(m)$ :

$$
\begin{aligned}
& I_{L}(m) \equiv I_{T T}+I_{V}+I_{S}, \\
& I_{T T}=\int d^{4} x\left[\pi^{T T} \dot{h}_{T T}-\left(\pi^{T T}\right)^{2}-\frac{1}{4}\left(\vec{\nabla} h^{T T}\right)^{2}-\frac{1}{4} m^{2}\left(h^{T T}\right)^{2}+\frac{1}{2} h_{T T^{T}} T^{T T}\right], \\
& I_{V}=\int d^{4} x\left\{-\left(2 \nabla^{2} \pi_{i}^{T}+T^{0}{ }_{i}\right) \dot{h}_{i}^{T}-2\left(\pi^{i T}{ }_{, k}\right)^{2}-\left(2 m^{2}\right)^{-1}\left[2 \nabla^{2} \pi_{i}^{T}+T^{0 i}\right]^{2}-\frac{1}{2} m^{2}\left(h_{i, j}^{T}\right)^{2}\right\}, \\
& -\frac{1}{3} I_{S}=\int d^{4} x\left[\chi \dot{h}^{T}+2 \chi^{2}+\frac{1}{8}\left(\nabla h^{T}\right)^{2}+\frac{1}{8} m^{2}\left(h^{T}\right)^{2}-\frac{1}{4} h^{T} T^{T}+\frac{1}{6} h^{T} T_{\alpha}^{\alpha}\right],
\end{aligned}
$$

where $\chi \equiv \pi^{i}{ }_{, i}$. The helicity-two part, $I_{T T}$, is (except for some instantaneous Newtonian interaction terms to be recovered below) identical in form to the linearized Einstein action $(m \equiv 0)$ plus a mass term and approaches it smoothly in the limit as $m \rightarrow 0$, since there are no further constraints on the variables. Note that it is in gauge-independent form; we could adjoin a new set of variables " $h_{0 \mu}$ ", etc., (not to be confused with the original ones) to put it into the covariant form (12) with $m=0$, or the form (26). Incidentally, it is easy to see that if $m=0$, the remaining parts [ (33c) and (33d)] just yield the necessary instantaneous terms and nothing else: For then $2 \nabla^{2} \pi_{i}^{T}+T^{0 i T}=0$ and $(33 \mathrm{c})$ reduces to $\left(\pi_{i, j}^{T}\right)^{2}$ which is now an instantaneous $\frac{1}{2} T^{0 i T} \nabla^{-2} T^{0 i T}$ term, while (33d) reduces to

$$
\frac{1}{2} T^{0 i T} \nabla^{-2} T^{0 i T}+\frac{3}{8} T^{0 i L} \nabla^{-2} T^{0 i L}-\frac{1}{8} T^{00} \nabla^{-2} T^{00}-\frac{1}{4} T^{00} \nabla^{-2} T_{i i}
$$

using $4 \nabla^{2} \chi+T^{0 i}{ }_{i}=0$ and $\nabla^{2} h^{T}+T^{00}=0$. These are just the right instantaneous interactions. For example, they combine with the effective $-\frac{1}{2} T_{i j}^{T T} \partial^{-2} T_{i j}^{T T}$ from helicity-two exchange in (33b) to yield the Einstein form

$$
\frac{1}{2}\left[T^{\mu \nu}\left(-\partial^{-2}\right) T_{\mu \nu}-\frac{1}{2} T_{\mu}^{\mu}\left(-\partial^{-2}\right) T_{\nu}^{\nu}\right]
$$

Returning to the $m \neq 0$ theory, the helicity-one part (33c) has explicit $m^{-2}$ dependence on the one hand, and is not in canonical, " $p \dot{q}-H$," form on the other. We will clearly need both a translation of the $\pi_{i}^{T}$ variable and a canonical transformation on $\left(\pi_{i}^{T}, h_{i}^{T}\right)$ to put the Hamiltonian in proper form. These operations will automatically eliminate the $m^{-2}$ as well. Define the new variables

$$
\begin{aligned}
\mathcal{E}_{i}^{T} & \equiv-2\left(-\nabla^{2} / m^{2}\right)^{-1 / 2} D^{-1 / 2} \pi_{i}^{T}-m^{-1} D^{1 / 2} T^{0 i T}, \\
A_{i}^{T} & \equiv m^{2}\left(-\nabla^{2} / m^{2}\right)^{1 / 2} D^{1 / 2} h_{i}^{T},
\end{aligned}
$$

where $-\nabla^{2}$ and $D \equiv\left(m^{2}-\nabla^{2}\right)^{-1}$ are positive. Then $I_{V}$ becomes

$$
\begin{aligned}
I_{V} & =\int d^{4} x\left[-\mathcal{E}_{i}^{T} \dot{A}_{i}^{T}-\frac{1}{2} \overrightarrow{\mathcal{E}}_{T}{ }^{2}-\frac{1}{4}\left(\vec{\nabla} \times \overrightarrow{\mathrm{A}}_{T}\right)^{2}-\frac{1}{2} m^{2} \overrightarrow{\mathrm{A}}_{T}{ }^{2}-A_{i}^{T}\left(-\nabla^{2} / m^{2}\right)^{-1 / 2} \mathfrak{D}^{1 / 2} T^{i j}{ }_{j}{ }^{T}\right]-\frac{1}{2} \int d^{4} x T_{0 i}^{T} \mathfrak{D} T_{0 i}^{T} \\
& \equiv I_{V}^{(0)}+I_{C}^{(1)} .
\end{aligned}
$$

The free part is precisely in gauge-invariant Maxwell form, as $m \rightarrow 0$, and the coupling to the transverse current $\left(T^{i j}{ }_{, j}\right)^{T}$ vanishes as $O(m)$, so that the helicity-one part becomes a free Maxwell field in the limit. The instantaneous term is the only matter-matter contribution from helicity-one exchange; its limit is $\frac{1}{2} T_{0 i}^{T}\left(-\nabla^{-2}\right) T_{0 i}^{T}$, the usual repulsive vector coupling. 
The final part, $I_{S}$, already has the form of a normal scalar field, in first-order language, to within the trivial rescaling:

$$
\tilde{h}=\frac{1}{2} \sqrt{3} h_{T}, \quad \tilde{\chi}=-2 \sqrt{3} \chi
$$

which gives

$$
I_{S}=\int d^{4} x\left[\tilde{\chi} \dot{\tilde{h}}-\frac{1}{2} \tilde{\chi}^{2}-\frac{1}{2}(\vec{\nabla} \tilde{h})^{2}-\frac{1}{2} m^{2} \tilde{h}^{2}\right]+\frac{1}{2} \sqrt{3} \int d^{4} x \tilde{h}\left(T^{T}-\frac{2}{3} T_{\alpha}^{\alpha}\right) .
$$

This represents a (nonghost) scalar field with coupling to $T_{\mu \nu}$ both through the spatial component $T^{T}$ and the four-scalar $T^{\alpha}{ }_{\alpha}$. We can translate away the former to leave a Lorentz-scalar expression plus instantaneous terms. This is easily done in second-order formalism, using the equality

$$
\begin{aligned}
T^{T} & \equiv T_{i i}-\nabla^{-2} T_{i j, i j} \\
& =T^{\alpha}{ }_{\alpha}+T^{00}-\nabla^{-2} T^{00}, 00 \\
& =T^{\alpha}{ }_{\alpha}+\nabla^{-2} \partial^{2} T^{00}
\end{aligned}
$$

so that

$$
I_{S}=\frac{1}{2} \int d^{4} x\left[-\left(\partial_{\mu} \tilde{h}\right)^{2}-m^{2} \tilde{h}^{2}\right]+(\sqrt{3} / 6) \int d^{4} x \tilde{h}\left(T_{\alpha}^{\alpha}+3 \nabla^{-2} \partial^{2} T^{00}\right) .
$$

Now translate: $\phi \equiv \tilde{h}+(\sqrt{3} / 2) \nabla^{-2} T^{00}$ to obtain, in the $m \rightarrow 0$ limit

$$
\begin{aligned}
I_{S} \underset{m \rightarrow 0}{\sim} \frac{1}{2} \int d^{4} x\left[-\left(\partial_{\mu} \phi\right)^{2}+(1 / \sqrt{3}) \phi T_{\alpha}^{\alpha}\right]+\frac{1}{8} \int d^{4} x T^{00}\left(\nabla^{-2} T_{\alpha}^{\alpha}{ }_{\alpha}-3 T^{T}\right) \\
\quad \equiv I_{S}^{(0)}+I_{C}^{(0)} .
\end{aligned}
$$

This is the action of a scalar field $\phi$ coupled to the Lorentz scalar $T_{\alpha}^{\alpha}$, together with two instantaneous "Coulomb" terms.

At this point, then, our total action has the limiting form

$$
I=\left[I_{T T}+I_{C}^{(1)}+I_{C}^{(0)}+I_{V}^{(0)}+I_{S}^{(0)}\right],
$$

where $I_{C}^{(1)}+I_{C}^{(0)}$ are the instantaneous terms in (37) and (39). The bracket is precisely the action of the $m=0$ system coupled to the conserved $T_{\mu \nu}$, entirely equivalent to the conventional covariant form (12) or (26). It would yield (as can also be checked directly from the present form) the retarded helicity-two interaction (35). The helicity-one part $I_{V}^{(0)}$ is a free Maxwell field, while $I_{s}^{(0)}$ represents a new Lorentz-invariant scalar system coupled to $T^{\alpha}{ }_{\alpha}$. One-scalar exchange then contributes the usual attractive form

$$
+\frac{1}{6} \int d^{4} x T_{\alpha}^{\alpha}\left(-\partial^{-2}\right) T_{\beta}^{\beta}
$$

which combines with (35) to replace the Einstein $\frac{1}{2}$ by $\frac{1}{3}$ in the trace-trace part. Because the helicity-zero part did not decouple from the source, we have a tensor-scalar theory in the limit, rather than a pure tensor. It may be compared with the linearization of the Brans-Dicke ${ }^{17}$ model, which conveniently parametrizes arbitrary tensorscalar mixtures by a coupling constant $\omega$. The present case corresponds to $\omega=0, \omega=\infty$ being the pure tensor limit. There is no corresponding connection though between the nonlinear massive and Brans-Dicke theories.

We have seen in this section that the limit as $m \rightarrow 0$ exists, for a conserved source. It is a tensor-scalar mixture, together with a free Maxwell field. The limit is smooth [although some local quantities, like the stress tensor of the massive field itself do have $\left(\mathrm{m}^{-2}\right)$ terms $\left.{ }^{4}\right]$. Any further scalar admixture will yield still larger deviations from the Einstein form [more attractive $\left(T_{\alpha}^{\alpha}\right)^{2}$ coupling], unless the scalar field has negative energy. In that case, all states would be unstable against radiation of negative scalar quanta. Although it is clear from the above that no acceptable (nonghost) admixture helps, one may also examine all possible mass terms ${ }^{12}$ by the same methods. One finds directly that either they lead to still worse discrepancies or contain ghosts. Finally, modification of the source cannot help either: For prescribed functions. $T^{\mu \nu}(x)$, one can, at the costs outlined in footnote 11, always arrange that $T^{\mu \nu}{ }_{, \nu}$ and $T^{\nu \mu}, \mu \nu$ be of $O\left(\mathrm{~m}^{2}\right)$ and compensate the scalar contribution. However, even this is not possible for a dynamical $T^{\mu \nu}$. We merely cite the simplest example here, a massive scalar field for which $T_{\alpha}^{\alpha}=-(\phi, \alpha)^{2}+2 M^{2} \phi^{2}$. No local addition of $O\left(m^{2}\right)$ to $T^{\mu \nu}$ will provide the desired interaction, as can easily be checked from the generalization of (17) for nonconserved $T^{\mu \nu}$ (Ref. 8): 


$$
\begin{gathered}
I_{\text {eff }} \sim \int d^{4} x\left(T^{\mu \nu} \Delta T_{\mu \nu}-\frac{1}{3} T^{\alpha}{ }_{\alpha} \Delta T_{\beta}^{\beta}+2 m^{-2} t_{\mu} \Delta t^{\mu}\right. \\
\left.+\frac{2}{3} m^{-2} T^{\alpha}{ }_{\alpha} \Delta t+\frac{2}{3} m^{-4} t \Delta t\right), \\
t^{\mu} \equiv T^{\mu \nu}{ }_{\nu}, \quad t \equiv t^{\mu}{ }_{, \mu}, \quad \Delta \equiv\left(-\partial^{2}+m^{2}\right)^{-1} .
\end{gathered}
$$

We conclude that the discontinuity is an unavoidable consequence ${ }^{18}$ of Lorentz-invariance and positive-energy requirements.

\section{FINITE - MASS EINSTEIN THEORY}

The addition of a mass to the Einstein theory is much more complicated than in the linearized theory. There, there is a unique choice of linear mass term which describes only a spin-two field. The full theory with zero mass being nonlinear, there is no reason to require the mass term to be linear. The meaning of a range or mass is defined in terms of an asymptotic falloff of forces or the propagation of weak excitations relative to a (minimum energy) background. Thus we think of it in terms of a Lorentz-invariant field theory, that is, as a term $m^{2} f^{\mu \nu}$ to be added to $G^{\mu \nu}$ in the Einstein equation. It is to be free of derivatives, and will of course break the gauge group (general coordinate invariance) of the massless theory. We also leave the coupling of matter $(\psi)$ to gravitation unchanged, so as to respect the observed equivalence principle. Then matter will respond to a given $g_{\mu \nu}$ as in the Einstein theory; it will move in an effective metric space, even though the dynamic $g_{\mu \nu}$ is no longer interpretable as a Riemannian metric because the gauge is broken. We then write the total action as

$$
I=\int d^{4} x\left[\mathcal{R}(g)+\mathcal{L}(\psi, g)-\frac{1}{4} m^{2} f(g)\right]
$$

with $f$ (and $f^{\mu \nu} \equiv \delta f / \delta g_{\mu \nu}$ ) to be determined further. At $m \equiv 0$, this is Einstein theory (which does not imply a smooth transition of the solution as $m \rightarrow 0$ ). A final general criterion is that since space-time is (experimentally) nearly Minkowskian it should still be possible to transform so that $g_{\mu \nu} \sim \eta_{\mu \nu}$, and to retain Lorentz invariance relative to $\eta_{\mu \nu}$.

We first show that $f$ cannot be a function of $g_{\mu \nu}$ alone, but must involve a background metric as an absolute object; the latter will have to be Lorentzinvariant. Let us dispose, as an example, of the cosmological term, $\lambda \sqrt{-g}$, as a mass term. It violates all our criteria: (1) it does not break gauge invariance, and hence does not increase the number of degrees of freedom of the gravitational field; (2) it forbids the existence of a stable matterfree Minkowski metric solution to the field equations (flat space as vacuum state); (3) it leads to the wrong mass term for small excitations. To see this, let us expand $\sqrt{-g}$ in terms of $h_{\mu \nu} \equiv g_{\mu \nu}$ $-\eta_{\mu \nu}$

$$
\sqrt{-g}=1+\frac{1}{2} h_{\alpha \alpha}-\frac{1}{2}\left(h_{\mu \nu} h^{\mu \nu}-\frac{1}{2} h_{\alpha \alpha}^{2}\right)+\cdots \cdot
$$

The linear term leads to a constant source in the Einstein equation (forbidding the $g=\eta$ solution), while the term is not of Pauli-Fierz form, but rather has a scalar-ghost contribution. The only other possible function of the metric alone is $f(-g)$, which has the form, near $\eta_{\mu \nu}$,

$$
\begin{aligned}
f(-g) \simeq & f(1)+\left[h^{\alpha}{ }_{\alpha}-\frac{1}{2} h_{\mu \nu} h^{\mu \nu}\right] f^{\prime}(\mathbf{1}) \\
& +\frac{1}{2}\left(h^{\alpha}{ }_{\alpha}\right)^{2}\left[f^{\prime}(\mathbf{1})+f^{\prime \prime}(\mathbf{1})\right] .
\end{aligned}
$$

The linear term vanishes only if $f^{\prime}(1)=0$, but then so does the $h_{\mu \nu} h^{\mu \nu}$ term, and the $\left(h^{\alpha}{ }_{\alpha}\right)^{2}$ can at most provide a mass for a scalar (but not the tensor) degree of freedom. Since $g_{\mu \nu} g^{\mu \nu}=1$, the deter minant is the only possible function of $g$ alone, and we must now have $f\left(g, g^{(0)}\right)$ where $g^{(0)}$ is a background metric. The most natural choice of background metric is $g_{\mu \nu}^{(0)}=\eta_{\mu \nu}$, the Minkowski metric of flat space since, locally, space is nearly flat. [More generally, $g^{(0)}$ should be a stable (minimum energy) solution of the source-free equations,

$$
G\left(g^{0}\right)+m^{2} \frac{\delta f}{\delta g_{\mu \nu}}\left(g^{0}, g^{0}\right)=0
$$

in order that the excitations $h_{\mu \nu} \equiv g_{\mu \nu}-g_{\mu \nu}^{(0)}$ of the theory have the solution $h_{\mu \nu}=0$. This is the case if $G\left(g^{0}\right)=0$, with $\delta f / \delta g_{\mu \nu}$ a power series in $h_{\mu \nu}$ with no constant term.] With the choice $\eta_{\mu \nu}, f$ must be such that flat space is a stable solution. This means that in the expansion of $f(\eta+h, \eta)$, there is no linear term in $h$ (or else $g=\eta$ is not a solution at all) and the quadratic term must have the Pauli-Fierz form,

$$
f(\eta+h, \eta) \simeq f(\eta)+h^{\mu \nu} h_{\mu \nu}-\left(h_{\alpha}^{\alpha}\right)^{2}+O\left(h^{3}\right) .
$$

Conversely, if $f$ is exactly in Pauli-Fierz form, the only solution $g_{\mu \nu}$ of the massive equations for which $f_{\mu \nu}(g)=0$ is the vacuum $g^{(0)}$. This provides an amusing Machian implication of the massive theory. While $h_{\mu \nu}=0$ is a solution, $h_{\mu \nu}=$ const $\times g_{\mu \nu}^{(0)}$ is not, for $G_{\mu \nu}(g)$ still vanishes; so must the mass term and this implies that the constant vanishes. Thus, vacuum $g=g^{(0)}$ is an isolated point. This is analogous to the property of massive electrodynamics that $\partial_{\nu} F^{\mu \nu}=0 \rightarrow A^{\mu}=0$; constant potentials are forbidden in the absence of charges.

To summarize, then, we take $f$ in what follows to be $f(g, \eta)$ such that $f \sim f(\eta)+h_{\mu \nu}{ }^{2}-\left(h^{\alpha}{ }_{\alpha}\right)^{2}+O\left(h^{3}\right)$. (Appendix B deals with a model violating this requirement.) We shall first use the Pauli-Fierz term itself, then consider an arbitrary function of the Pauli-Fierz term, $f\left(h_{\mu \nu} h^{\mu \nu}-\left(h_{\alpha}^{\alpha}\right)^{2}\right)$; in both 
cases the $m \rightarrow 0$ limit is unacceptable. We have not been able to solve the constraints for more general quadratically correct mass terms; hence we have not been able to prove that there is no mass term for which the $m \rightarrow 0$ limit exists. On the other hand, the requirement that the vacuum be a stable equilibrium already assures us that the experimental difficulties of the linearized theory will persist in the full theory, even if some mass term can be found for which the $m \rightarrow 0$ limit exists. ${ }^{19}$

For simplicity, we omit the coupling to matter in this part of our discussion. The modifications are very simple, since the usual dynamical matter systems may be put in the form

$$
I_{m}=\int d^{4} x\left(p \dot{q}-N \theta^{0}-N_{i} \theta^{i}\right)
$$

where the $\theta^{\mu}$ depend on the dynamical variables $(p, q)$ and $g_{i j}$. This means that the $\theta^{\mu}$ are just to be added to the gravitational $R^{\mu}$ in in what follows. But this has unpleasant consequences also for the matter field equations since the Hamiltonian of Eq. (49) below is now that of gravitation plus matter, both of which will be shown to diverge as $1 / m^{2}$ in the limit. These difficulties for matter arise only when the dynamics of the $g_{\mu \nu}$ are taken into account; for a given external $g_{\mu \nu}$, matter still obeys the equivalence principle since it is coupled to $g_{\mu \nu}$ according to the usual covariant prescription.

Expressed in terms of the $\left(g_{i j}, N, N_{i}\right)$ notation, the Pauli-Fierz mass term has the form

$$
\begin{aligned}
f & \equiv h_{\mu \nu} h^{\mu \nu}-\left(h_{\alpha}^{\alpha}\right)^{2} \\
& =h_{i j} h^{i j}-\left(h_{i i}\right)^{2}+2 h_{i i} h_{00}-2\left(h_{0 i}\right)^{2} \\
& =\left(h_{i j}\right)^{2}-h^{2}-2 N_{i}{ }^{2}+2 h\left(1-N^{2}-N_{i} g^{i j} N_{j}\right), \\
h & \equiv h_{i i} .
\end{aligned}
$$

In contrast to the linear case, it is now quadratic in $N$, so that neither $N$ nor $N_{i}$ are Lagrange multipliers, but are determined by the constraint equations their variations imply. This conclusion, that there are now six rather than five degrees of freedom, is totally independent of the particular mass term - ghost or not. It is a consequence of Lorentz invariance alone, which excludes linearity in $N$ or $N_{i}$ (the only exception is the irrelevant cosmological term $\sqrt{-{ }^{4} g} \equiv N \sqrt{g}$ ). The constraint equations obtained by varying the $N$ 's are:

$$
\begin{aligned}
& R^{0}=m^{2} h N, \\
& m^{2}\left(\eta^{i j}-h g^{i j}\right) N_{j}=R^{i} .
\end{aligned}
$$

The Hamiltonian obtained by eliminating the $N^{\prime} \mathrm{s}$ in the action is then

$$
\begin{gathered}
H=\frac{1}{2} \int d^{3} r\left\{\left[\left(R^{0}\right)^{2} h^{-1}+R^{i}\left(\eta^{i j}-h g^{i i}\right)^{-1} R^{j}\right] m^{-2}\right. \\
\left.+\frac{1}{2} m^{2}\left[h_{i j}{ }^{2}-h^{2}+2 h\right]\right\} .
\end{gathered}
$$

Aside from the mass term, it is entirely of $O\left(\mathrm{~m}^{-2}\right)$. There are no further constraints among the six pairs $\left(\pi^{i j}, g_{i j}\right)$ of conjugate variables, except for nonsingularity requirements on existence of the inverses $g^{i j}, g^{00}$, etc. In particular, $0<\left(m^{2} h\right)^{-1} R^{0}$ and, for localized sources and excitations, the boundary conditions $R^{0}-m^{2} h \rightarrow 0, \pi^{i j},{ }_{j} \rightarrow 0$ hold at spatial infinity where all amplitudes tend to zero. The first condition is a relic of the linearized theory, where it holds everywhere, eliminating the sixth degree of freedom. ${ }^{20}$

Another rather general conclusion bears on the nonexistence of the $m \rightarrow 0$ limit. The over-all explicit $m^{-2}$ dependence of the unconstrained Hamiltonian (49) is essentially independent of details of the mass term, being traceable to the $N R$ form of the massless action. This original "vanishing Hamiltonian" term is associated with general coordinate invariance and is peculiar to this particular gauge theory. The limit will only exist, therefore, if $m^{-2}$ can be scaled away, but the scaling must respect the $\pi^{i j} g_{i j}$ term (be a canonical transformation). The specific dependence of $R^{0}$ and $R^{i}$ on $\pi^{i j}$ and $g_{i j}$, as given in (25) makes it unlikely that a suitable rescaling of either term exists, although we have no formal proof to this effect. ${ }^{21}$

Perhaps still more unpleasant is the fact that the energy (49) has no lower bound, so that the system is unstable and the sixth degree of freedom leads to ghost properties for any $m$. Consider, for simplicity, a state which is characterized at a given instant by $R^{i}=0$, and excitations with small negative $h$. The mass term can be made negligible, while the energy

$$
H \sim-m^{-2} \int d^{3} r\left(R^{0}\right)^{2}|h|^{-1}
$$

can be made arbitrarily large and negative for fixed $m$, compatible with the boundary conditions.

It might be thought that this is due to the fact that the Pauli-Fierz mass term is not positive definite in its original form; it is possible to analyze the general class of mass terms $f(\chi)$, $\chi \equiv h^{\mu \nu} h_{\mu \nu}-\left(h_{\alpha}^{\alpha}\right)^{2}$ subject only to the restriction $f^{\prime}(0)=1$ so that the quadratic terms are correct. Then, we find that either the same instability persists or that no solutions exist as $m^{2} \rightarrow 0$, i.e., the metric becomes complex. To see this, we observe that the equations for $N$ and $N_{i}$ become, with the new mass term 


$$
\begin{aligned}
& R^{0}=m^{2} h N f^{\prime}(\chi), \quad R^{i}=\left(\eta^{i j}-h g^{i j}\right) N_{j} m^{2} f^{\prime}(\chi), \\
& \chi=h_{i j} h_{i j}-h^{2}+2 h-2\left(R^{0}\right)^{2} m^{-4} h^{-1}\left[f^{\prime}(\chi)\right]^{-2}-2 R^{i}\left(\eta^{i j}-h g^{i j}\right)^{-1} R^{j} m^{-4}\left[f^{\prime}(\chi)\right]^{-2}
\end{aligned}
$$

and the Hamiltonian becomes

$$
H=\int d^{3} r\left\{\frac{1}{4} m^{2} f(\chi)+\left(R^{0}\right)^{2} m^{-2} h^{-1}\left[f^{\prime}(\chi)\right]^{-1}+R^{i}\left(\eta^{i j}-h g^{i j}\right)^{-1} R^{j} m^{-2}\left[f^{\prime}(\chi)\right]^{-1}\right\} .
$$

The mass term $f(\chi)$ must be bounded from below; otherwise, by taking $R^{0}=0=R^{i}$, the Hamiltonian density may be taken to $-\infty$. Thus, $\chi f^{\prime}(\chi) \geqslant 0$ as $|\chi| \rightarrow \infty$. Now, let $h$ become small and negative with $R^{i}=0$ and fixed $R^{0}$ and assume $|f(\chi)| \rightarrow F|\chi|^{\alpha}, \alpha>\frac{1}{2}$, then

$$
\chi \underset{h \rightarrow 0-}{\sim}|h|^{-1 /(2 \alpha-1)}\left[2\left(R^{0}\right)^{2} / \alpha^{2} m^{4} F^{2}\right]
$$

and the Hamiltonian density is

$$
\sim|h|^{-\alpha /(2 \alpha-1)}\left[\left(R^{0}\right)^{2} / m^{2} F_{\alpha}\right]\left\{(2 \alpha)^{-1}\left[2\left(R^{0}\right)^{2} / m^{4} \alpha^{2} F^{2}\right]^{\alpha-1}-\left[2\left(R^{0}\right)^{2} / m^{2} \alpha^{2} F^{2}\right]^{-(\alpha+1)}\right\} .
$$

For $1>\alpha>\frac{1}{2}$ and $R^{0}$ sufficiently large compared to $\alpha^{2} m^{4} F^{2}$, for $\alpha>1$ and $R^{0}$ sufficiently small, or for $\alpha=1$ and any $R^{0}$, the energy density goes to $-\infty$.

In the case where $\alpha<\frac{1}{2}$, there is no solution to Eqs. $\left(48^{\prime}\right)$ as $h$ becomes small; if we assume $\chi \rightarrow \infty$, we obtain the contradiction $\chi \sim|h|^{-1 /(2 \alpha-1)}()$, but if we assume $\chi$ finite in $f^{\prime}(\chi)$, we obtain $\chi \sim 1 /|h|$.

Hence no asymptotic behavior of $f$ produces an acceptable theory; either, (a) the energy density is not bounded from below or (b) the equations do not have a solution for $h \sim 0-$.

A proof for arbitrary mass terms appears to be rather difficult because we cannot solve the constraints when arbitrary powers of $N$ and $N_{i}$ are present. It should be emphasized that there is nothing pathological about the excitations needed to obtain a negative energy; for example, helicity two amplitudes will accomplish this when modulated by small negative excitations of the trace $h$.

We have not yet discussed the massive theory in terms of the Lorentz covariant form of the field equations,

$$
g^{\mu \nu}(g)+\frac{1}{4} m^{2} f^{\mu \nu}(g, \eta)=\tau^{\mu \nu}(\psi, g),
$$

where $f^{\mu \nu}$ is the derivative of the mass term with respect to $g_{\mu \nu}$. For any dynamical source, $\tau^{\mu \nu}(\psi)$ is automatically covariantly conserved by virtue of the $\psi$ field equations alone..$^{22}$ Likewise $g^{\mu \nu}$ is identically conserved: $\mathcal{G}^{\mu \nu}{ }_{i \nu} \equiv 0$. This means that the covariant divergence of $f^{\mu \nu}$ considered as a formal contravariant tensor density must vanish, and therefore also that $\partial_{\mu}\left(f_{; \nu}^{\mu \nu}\right)=0$. The vanishing of the divergence provides, as in linearized theory, the four conditions to remove the vector and one of the scalar fields present in an arbitrary 10component $h_{\mu \nu}$. The trace of (51) implied that

$$
-\mathbb{R}+\frac{1}{4} m^{2} g_{\mu \nu} f^{\mu \nu}=\tau^{\alpha}{ }_{\alpha} .
$$

Unlike the linear situation, however, $f_{; \mu \nu}^{\mu \nu}$ is not the same as $R$, being a noninvariant quantity. The two differ by nonlinear terms, which cannot be transformed away at a point because coordinate invariance is not available. More important, this difference involves second derivative terms so that the trace condition (52) is no longer a constraint as it was in the linear case: This is where the sixth degree of freedom arises from this point of view, since only the four divergence conditions are still constraints.

It is not possible to find a propagator form for the equation (50) with or without mass. ${ }^{23}$ However, we can find an expression for effective interactions due to one-graviton exchange as follows: We write the Einstein equations (in units $K=1$ ) as

$$
G_{\mu \nu}^{L}+\frac{1}{4} m^{2} f_{\mu \nu}^{L}=-G_{\mu \nu}^{\mathrm{NL}}+\tau_{\mu \nu}(\psi, g),
$$

where $G^{L}$ and $G^{\mathrm{NL}}$ are the parts of $\subseteq$ and which are respectively linear and nonlinear in $h$, and $\tau^{\mu \nu}(\psi, g)$ is the matter stress tensor. We take a linear mass term $f^{L}$ (for simplicity). In the absence of mass $-G_{\mu \nu}^{\mathrm{NL}}$ is in fact the symmetric stress tensor of the Einstein field, treated as a Lorentz-covariant system. ${ }^{7}$ In particular, the total stress tensor of the system, $-G_{\mu \nu}^{\mathrm{NL}}+\widetilde{T}_{\mu \nu}$, is (ordinarily) conserved by virtue of the field equation and the covariant conservations of $G_{\mu \nu}$ and $\tau_{\mu \nu}(\psi, g)$.

Thus for $m \equiv 0$, the Einstein equations read

$$
G_{\mu \nu}^{L}=\left[\mathcal{T}_{\mu \nu}(\psi, g)-G_{\mu \nu}^{\mathrm{NL}}\right] \equiv \mathcal{T}_{\mu \nu}^{\mathrm{tot}}
$$

For the massive case, the coupled system is still conservative, but the conserved total stress tensor must now include the contribution $T_{\mu \nu}^{m}$ from the mass part of the action (the theory of Freund et $a l .^{24}$ has the property that the mass term $m^{2} f^{\mu \nu}$ is just $T_{\mu \nu}^{m}$; it is discussed in detail in Appendix B); $-G_{\mu \nu}^{N L}$ is still the contribution from the $m \equiv 0$ part. That is,

$$
\begin{aligned}
G_{\mu \nu}^{L}+\frac{1}{4} m^{2} f_{\mu \nu}^{L} & =\mathcal{T}_{\mu \nu}^{\text {tot }}-T_{\mu \nu}^{m} \\
& \equiv T_{\mu \nu}^{0},
\end{aligned}
$$




$$
\partial_{\mu} T_{\mu \nu}^{\mathrm{tot}}=0
$$

These are the linear massive equations with a nonconserved source. The nonconserved part is formally proportional to $m^{2}$, since $\tau^{\mu \nu}, v \sim m^{2} h h$. The effective source-source interaction linear in $G$ is given by (42), which is valid for any nonconserved $\tau^{\mu \nu}$. In our case $T_{m, \nu}^{\mu \nu}=-\tau^{\mu \nu}{ }_{, \nu}$, so the $m^{-2}$ factors cancel in (42). One could also iterate in $h$, but this would give higher powers of $G$. We can therefore conclude that the one-graviton exchange interaction for weak excitations and matter sources $\mathscr{T}^{\mu \nu}(\psi)$ agrees with that of the massive linearized theory and so disagrees, for small $m$, with the linearized Einstein prediction. This does not mean that the full theory has a $m \rightarrow 0$ limit, of course, since already at the first iteration quantities such as $h_{\alpha \alpha} \sim m^{-2} \tau$ enter. Conceivably, all inverse powers of $m$ collect into "gauge" quantities and it might then happen further that the interaction remaining coincides with the Einstein result in the limit. No sign of such an occurrence is found in next order (in $G$ ) calculations.

A final remark concerns the replacement of the absolute element $\eta_{\mu \nu}$ by a dynamical field $f_{\mu \nu}$. The resulting theory has quite different motivation and scope from the single massive field under investigation here. The two-tensor model has the action $^{25,26}$

$$
I=\int d^{4} x\left[R(g)+\frac{1}{4} m^{2} f(f, g)+\mathcal{R}(f)\right]
$$

For $m \equiv 0$, we have two decoupled Einstein theories each with 2 degrees of freedom. In linear approximation, one finds a sum of massive plus massless spin-two fields upon diagonalizing. In the full case, there is a general coordinate invariance under simultaneous tensor transformation of $g_{\mu \nu}$ and $f_{\mu \nu}$, which should reduce the $a$ priori 12 degrees of freedom to 8 . To the extent that a diagonalization is possible here, this would indicate that the massive system still has 6 (rather than 5 ), and "diagonalized gravitation," 2 degrees of freedom.

\section{CONCLUSION}

Experimental evidence for a cutoff of gravitational forces would present a major theoretical problem if our contention that general relativity has no acceptable neighbors is correct. In any case, we must conclude that the requirement of continuity in theoretical models and fundamental invariance principles cannot always be upheld simultaneously.

\section{APPENDIX A}

In dealing with the linear theory, we have used the constraint equations to eliminate the "natural" redundant variables $N_{i}$ and the gauge part $h^{L}$ of the metric, in terms of $\pi^{i}$ and $h^{T}$. This choice of variables, which gave rise to the $\mathrm{m}^{-2}$ factors in the reduced action is, of course, not unique. We illustrate here how the results are unchanged by the "opposite" choice of constraint variables, in which we solve Eqs. (29) as follows:

$$
2 \pi^{i j}{ }_{, j}=-T^{0 i}+m^{2} N_{i}, \quad h^{T}=\mathfrak{D}\left(T^{00}-2 m^{2} \nabla^{2} h^{T}\right)
$$

in contrast to (31) and (32). The tensor $\left(I_{T T}\right)$ part of the action is unchanged, of course. The vector part becomes

$$
\bar{I}_{V}=m^{2} \int\left[N_{i}^{T} \dot{h}_{i}^{T}+\frac{1}{2} N_{i}^{T} m^{2} \nabla^{-2} N_{i}^{T}+\frac{1}{2} h_{i}^{T} \nabla^{2} h_{i}^{T}-\frac{1}{2}\left(N_{i}^{T}\right)^{2}+N_{i}^{T} \nabla^{-2} T^{0 i}\right]+\frac{1}{2} \int T^{0 i T} \nabla^{-2} T^{0 i T}
$$

which requires the rescaling

$$
\overline{\mathcal{E}}_{i}^{T}=m\left(-\nabla^{2} \mathfrak{D}\right)^{-1 / 2} N_{i}^{T}, \quad \bar{A}_{i}^{T}=m\left(-\nabla^{2} D\right)^{1 / 2} h_{i}^{T}
$$

to bring it to the canonical form

$$
\bar{I}_{V}=\int\left\{\overline{\mathcal{E}}_{i}^{T} \dot{\bar{A}}_{i}^{T}-\frac{1}{2} \overline{\mathcal{E}}_{i}^{T 2}-\frac{1}{2} \bar{A}_{i}^{T} \mathscr{D}^{-1} \bar{A}_{i}^{T}-m \overline{\mathcal{E}}_{i}^{T}\left[1-\nabla^{2} D\right]^{1 / 2} T^{0 i} T\right\}+\frac{1}{2} \int T^{0 i T} \nabla^{-2} T^{0 i T}
$$


(Note that in this choice, the problem is one of $m^{+2}$ rather than $m^{-2}$.) The scalar part is rather complicated, but reduces to the following form in terms of

$$
\bar{\phi}=m^{2} \sqrt{3} \nabla^{2} D h^{L}, \quad \bar{\pi}=\frac{1}{2} m^{2} \sqrt{3} N^{L}
$$

as $m \rightarrow 0$

$$
\begin{aligned}
\bar{I}_{S}= & -\int\left[\bar{\pi} \dot{\bar{\phi}}+\frac{1}{2} \bar{\pi}^{2}+\frac{1}{2}(\vec{\nabla} \bar{\phi})^{2}\right] \\
& -\frac{1}{2}(3)^{-1 / 2} \int \bar{\phi} T_{\alpha}^{\alpha} \\
& -\frac{1}{2} \int T^{00} \nabla^{-2}\left(T^{00}+\frac{1}{2} T^{T}-\frac{1}{4} \nabla^{-2} T^{i j}{ }_{i j}\right) .
\end{aligned}
$$

The resulting limit $m \rightarrow 0$ is identical to that in the text, namely the vector part decouples according to (A3), the scalar field maintains the same coupling to $T_{\alpha}{ }^{\alpha}$, and a set of instantaneous direct $T^{\mu \nu}-T^{\lambda \sigma}$ couplings which together yield the effective interaction (17).

In this treatment, the $\mathrm{m}^{-2}$ factor in the Hamiltonian does not appear, but the full action, including the $p \dot{q}$ term, is multiplied by $m^{2}$. We require that the theory be expressed in terms of canonical variables scaled so that the energy (in the $m \rightarrow 0$ limit) is finite for finite values of the variables. This rescaling restores the form of the effective interaction given in Eq. (17). In the nonlinear theory, no such rescaling is possible and the energy does not remain finite as $m \rightarrow 0$ for any choice of canonical variable.

\section{APPENDIX B}

It is of interest, both for its own sake and to illustrate the physical requirements we impose on finite-range models, to consider one version of a "ghost" theory in detail. The most appealing model is the one which carries over from general relativity the property that the source of the linearized field is the full stress tensor of the field itself. ${ }^{24}$ The mass term then takes on the form

$$
4 f(\eta, g)=\left(\frac{1}{2} \eta_{\mu \nu} g^{\mu \nu}-1\right) \sqrt{-g}
$$

whose linearized part is

$$
f \multimap-\frac{1}{4} m^{2}\left[h_{\mu \nu} h_{\mu \nu}-\frac{1}{2}\left(h_{\alpha}^{\alpha}\right)^{2}\right]
$$

and has a negative-energy massive scalar component.

A different origin may be given for this mass term as follows. Consider a massless scalar field

$$
\mathcal{L}(\phi)=-\frac{1}{2} \phi_{, \mu} \phi_{, \nu} g^{\mu \nu} \sqrt{-g}+\frac{1}{2}\left(\mu^{2} \phi^{2}-\frac{1}{2} \lambda^{2} \phi^{4}\right) \sqrt{-g} .
$$

This term has a constant equilibrium solution (in the absence of gravitation) $\phi^{2}=\phi_{0}^{2}=\mu^{2} / \lambda^{2}$ which, by the "Higgs" phenomenon, ${ }^{27}$ imparts a mass to long-range fields (e.g., electromagnetic) to which it couples. In the same way, this state yields a cosmological (rather than mass) term $\sim \frac{1}{4}\left(\mu^{4} / \lambda^{2}\right) \sqrt{-g}$ for gravitation. However, if we generalize this idea to vacuum solutions in which simultaneously

$$
\left\langle\phi^{2}\right\rangle \equiv \mu^{2} / \lambda^{2}
$$

and

$$
\left\langle\phi_{, \mu} \phi, \nu\right\rangle=\mu^{2 \frac{1}{4}} \eta_{\mu \nu}\left\langle\phi^{2}\right\rangle,
$$

we obtain precisely (B1), i.e., a real mass contribution also to gravitation.

Let us now write the total action in the $N, N_{i}$ form, using the fact that ( $g^{i j}$ and $\sqrt{g}$ now refer to the three-dimensional metric)

$$
\begin{aligned}
f(g, \eta) & \equiv \sqrt{-{ }^{4} g}\left[\frac{1}{2} \eta_{\mu \nu}{ }^{4} g^{\mu \nu}-1\right] \\
& =N \sqrt{g}\left(\frac{1}{2} \eta_{i j} g^{i j}-1\right)-\frac{1}{2} N^{-1} \sqrt{g}\left(N^{i} \eta_{i j} N^{j}-1\right) .
\end{aligned}
$$

The action then has the form

$$
\begin{aligned}
I=\int d^{4} x[ & \pi^{i j} \dot{g}_{i j}-N \bar{R}^{0}-N_{i} R^{i} \\
& \left.+\frac{1}{2} m^{2} \sqrt{g} N^{-1}\left(N^{i} \eta_{i j} N^{j}-1\right)\right], \\
\bar{R}^{0} \equiv R^{0}+ & m^{2} \sqrt{g}\left(\frac{1}{2} g^{i j} \eta_{i j}-1\right) .
\end{aligned}
$$

The resulting constraints may be solved to yield

$$
\begin{aligned}
& N^{2}=m^{4} g\left(2 m^{2} \sqrt{g} \bar{R}^{0}+R^{i} \eta_{i j} R^{j}\right)^{-1}, \\
& N^{i}=R_{i}\left[2 m^{2} \sqrt{g} \bar{R}^{0}+R^{i} \eta_{i j} R^{j}\right]^{-1 / 2}
\end{aligned}
$$

Inserting these into the action yields the Hamiltonian form (in terms of 6 degrees of freedom),

$$
\begin{aligned}
& I=\int\left(\pi^{i j} g_{i j}-H\right), \\
& H \equiv\left(2 m^{2} \sqrt{g} \bar{R}^{0}+R^{i} \eta_{i j} R^{j}\right)^{1 / 2},
\end{aligned}
$$

which appears to be positive definite. Since in addition, the linearized approximation here corresponds to a scalar-ghost admixture, and so gives the linearized Einstein interaction in the weak-field limit, ${ }^{13}$ it would seem that this model has at least two improvements over that in text: Its energy is positive and it has correct linearized behavior. However, it is unacceptable: The vacuum is not a local minimum, but only a saddle point, as may be seen by considering equilibrium (static) configurations, or simply expanding $H$ to quadratic order, where it is found to agree with the linearized (ghost) version $H$. That for appropriate excitations the quadratic part of $H$ can be negative may seem irrelevant in view of the 
apparent positivity of the complete $H$ of Eq. (B8). Unfortunately, the argument of the square root is not intrinsically positive (except for the $R_{i}{ }^{2}$ term), even though its positivity is required for the theory to make sense, i.e., for $N^{2}$ to be positive (B7) (otherwise, the effective Riemannian metric "seen" by matter will become pathological). Therefore one would have to impose that the excitations respect this requirement, i.e., cut off arbitrarily those modes which take $H$ below its vanishing vacuum $(g=\eta)$ state value. Instability near vacuum $(g \simeq \eta)$ is the reason for rejecting this and other models whose quadratic mass is not of Pauli-Fierz form. The variation of $h_{\mu \nu}$ yields only one extremum, $h_{\mu \nu}=0$; thus the system will not, of itself, find a stable vacuum for any physical metric.

\section{APPENDIX C}

The ghost properties of all but the Pauli-Fierz model are easily evidenced as follows. A general symmetric tensor $h_{\mu \nu}$ has 10 components corresponding to spin-2, spin-1, and two spin-0 fields.
The four-vector part of $h_{\mu \nu}$ is a spin-1 plus spin-0 mixture and always yields ghosts (like the scalar part of $A_{\mu}$ in a vector theory). If one removes this from the start, the most general equation is like Eq. (13) with an arbitrary coefficient $\alpha$ of $\eta_{\mu \nu} h^{\sigma}{ }_{\sigma}$ in the mass term. ${ }^{16}$ Using the divergence and trace as in text, one obtains (with a conserved $T^{\mu \nu}$ for simplicity),

$$
\left[-(1-\alpha) \partial^{2}+m^{2}\left(2 \alpha-\frac{1}{2}\right)\right] h_{\sigma}^{\sigma}=-\frac{1}{2} T_{\sigma}^{\sigma}
$$

which means that $\alpha$ must satisfy $1 \geqslant \alpha \geqslant \frac{1}{4}$ for causality. Further, unless $\alpha=1, h^{\sigma}{ }_{\sigma}$ becomes a scalar degree of freedom rather than a constraint variable, and one obtains the solution for $h_{\mu \nu}$ as

$$
\begin{aligned}
h_{\mu \nu}= & \Delta\left(m^{2}\right)\left(T_{\mu \nu}-\frac{1}{3} \eta_{\mu \nu} T_{\sigma}^{\sigma}\right) \\
& -\eta_{\mu \nu} \frac{1}{6} \Delta\left(m^{2}(4 \alpha-1) / 2(1-\alpha)\right) T_{\sigma}^{\sigma} .
\end{aligned}
$$

Thus, for every $m \neq 0$, there is a scalar ghost. However, if we take the $m \rightarrow 0$ limit anyway, we find $h_{\mu \nu} \rightarrow D\left(T_{\mu \nu}-\frac{1}{2} \eta_{\mu \nu} T_{\sigma}^{\sigma}\right)$, which agrees with the massless theory.
*Work supported in part by the U. S. Atomic Energy Commission under AT(45-1)-1388B.

†Work supported in part by U.S.A.F. under Grant No. OSR 70-1864.

\$ Permanent address.

${ }^{1}$ E. Schrödinger and L. Bass, Proc. Roy. Soc. (London) A232, 1 (1955).

${ }^{2}$ E. C. G. Stueckelberg, Helv. Phys. Acta 30, 209

(1957); D. G. Boulware and W. Gilbert, Phys. Rev. 126, 1563 (1962); S. Deser, Ann. Inst. Henri Poincaré 16, 79 (1972); A. S. Goldhaber and M. M. Nieto, Rev. Mod. Phys. 43, 277 (1971).

${ }^{3}$ H. Van Dam and M. Veltman, Nucl. Phys. B22, 297 $(1970)$; L. D. Faddeev and A. A. Slavnov, Theor. Math. Phys. $\underline{3}, 18$ (1970); S. K. Wong, Phys. Rev. D $\underline{3}, 945$ (1971).

${ }^{4} \mathrm{~S}$. Deser (unpublished).

${ }^{5}$ See also Y. Iwasaki, Phys. Rev. D 2 , 2255 (1970), and V. I. Zaharov, ZhETF Pis. Red. 12, $\overline{4} 47$ (1970) [JETP Lett. 12, $312(1970)]$.

${ }^{6}$ G. A. Seielstad, R. A. Sramek, and K. Weiler, Phys. Rev. Letters 24, 1373 (1970); D. O. Mu, R. D. Ebers, and E. B. Fomalont, ibid. 24, 1377 (1970).

${ }^{7}$ See S. Deser, J. Gen. Rel. Grav. 1, 9 (1970), where earlier references are also given.

${ }^{8} \mathrm{~J}$. Schwinger, Particles, Sources and Fields (AddisonWesley, Reading, Massachusetts, 1970), p. 78

${ }^{9} \mathrm{We}$ use the metric with signature $(-1,1,1,1)$ and units in which $\hbar=1=c$.

${ }^{10}$ Just as photons can only couple to conserved currents, a zero-mass spin-two particle can only couple to a con- served second-rank tensor; see S. Weinberg, Phys. Rev. 134, B882 (1964).

II It is possible to take the stress tensor to be conserved only in the limit $\partial_{\nu} T^{\mu \nu} \sim O\left(\mathrm{~m}^{2}\right)$, thus avoiding the discontinuity at the limit. This is, however, at the cost of using stress tensors which possess explicit singularities in $p^{2}$; if these singularities are extracted from $T$ and included in the propagator, they change the limit only if they correspond to ghost excitations. Furthermore, the singularities, viewed as a part of the stress tensor, imply, as the limit is taken, a nonlocalized stress tensor. An example is $T^{\mu \nu}=T_{0}^{\mu \nu}+\frac{1}{2} \eta^{\mu \nu} m^{2}\left(p^{2}+\alpha m^{2}\right)^{-1} T_{0 \lambda}^{\lambda}$, where $T_{0}^{\mu \nu}$ is the conserved stress tensor in the limit. If the interaction is written in terms of $T_{0}$, it becomes, for pure spin-two exchange by $T$,

$$
\begin{aligned}
& \left(p^{2}+m^{2}\right)^{-1}\left(T_{0}^{\mu \nu} T_{0 \mu \nu}-\frac{1}{3} T_{0 \lambda}^{\lambda} T_{0 \sigma}^{\sigma}\right) \\
& -\frac{1}{3}\left(p^{2}+\alpha m^{2}\right)^{-1} T_{0 \lambda}^{\lambda} T_{0 \sigma}^{\sigma} \\
& +\frac{1}{6}\left(p^{2}-2 m^{2}\right)\left(p^{2}+\alpha m^{2}\right)^{-2} T_{0 \lambda}^{\lambda} T_{0 \alpha}^{\alpha} \\
& \underset{m \rightarrow 0}{\sim} p^{-2}\left(\boldsymbol{T}_{0}^{\mu \nu} T_{0 \mu \nu}-\frac{1}{2} T_{0 \mu}^{\lambda} T_{0 \nu}^{\nu}\right)
\end{aligned}
$$

but has, before $m \rightarrow 0$, a ghost pole and a double pole. See also the discussion preceding Eq. (42).

${ }^{12}$ W. Pauli and M. Fierz, Proc. Roy. Soc. (London) $\mathrm{A} 73,211$ (1939).

${ }^{13} \mathrm{~A}$ catalog of possible admixtures is given by V. I. Ogievetsky and I. V. Polubarinov, Ann. Phys. (N.Y.) 35, 167 (1965). 
${ }^{14}$ One can obtain the form of $S_{\mu \nu}$ by arguments analogous to those used in discussing the one-graviton exchange; $h_{\text {out }}=h_{\text {in }}+\Delta S$, then $S_{\mu \nu, \nu}=0=S_{\alpha}^{\alpha}$ to keep the in and out fields pure spin two. If we expand,

$$
S^{\mu \nu}=\boldsymbol{T}^{\mu \nu}+\alpha \eta^{\mu \nu} \boldsymbol{T}_{\lambda}^{\lambda}+\beta \partial^{\mu} \partial^{\nu} \boldsymbol{T}_{\lambda}^{\lambda},
$$

the form given is the unique solution, for $T^{\mu \nu}, \nu=0$. We also remark here that in terms of action at a distance, any coefficient of $\left(T^{\alpha}{ }_{\alpha}\right)^{2}$ is acceptable - it is only in terms of the underlying field theory that compensations cannot be made consistently.

${ }^{15}$ R. Arnowitt, S. Deser, and C. W. Misner, Phys. Rev. 117, 1595 (1960).

${ }^{16} \mathrm{~A}$ pure $m^{2} h^{2}$ term corresponds to the massless field in the gauge $h=0$.

${ }^{17}$ C. Brans and R. H. Dicke, Phys. Rev. 124, 925 (1961).

${ }^{18} \mathrm{Of}$ course, if the new scalar degree of freedom is arbitrarily frozen by insisting that $4 \nabla^{2} \chi+T^{0 i}, i=0$ and $\nabla^{2} h^{T}+T^{00}=0$, then the scalar action becomes an instantaneous interaction which leads to the Einstein limit. This is not surprising because the freeze is just a way of writing the massless theory in a peculiar gauge.

${ }^{19} \mathrm{In}$ a recent paper, A. I. Vainshtein, Phys. Letters 39B, $393(1972)$, argues that a physically continuous solution in the $m=0$ limit may be obtained by performing a coordinate transformation so that the transformed metric limits smoothly to the Schwarzschild metric. The coordinate transformation is conjectured to be a nonanalytic function of $G$. A lowest-order calculation bears this out but, upon iteration the Schwarzschild metric functions go as $m^{2} \sqrt{M G} \rho^{3 / 2}$ and it is not obvious that the functions are well behaved at $\infty$.

If the metric is asymptotically equal to the background metric, then the solution exhibited in Eq. (22) must hold for large $r$ since this is the unique solution to the asymptotic field equations. Thus, for the conjecture to hold, the coordinate transformation cannot go to zero at infinity and the background metric must be unrelated to the asymptotic metric for any nonzero total energy.

${ }^{20}$ The linear massive theory may be recovered from the full action by expanding $I$ to quadratic order and systematically using this condition.

${ }^{21} \mathrm{~A}$ finite limit is but one requirement; the limiting Hamiltonian must also make sense physically. Thus, even if some nonlinear gauge choice succeeds in removing the $\mathrm{m}^{-2}$ singularities, the resulting theory is not guaranteed to be physical. We also reject the arbitrary freezing (by setting $R^{\mu}=0$ ) of the new variables, whose excitations are allowed by the dynamics.

${ }^{22}$ The variation of $I_{m}(\psi, g)$ under infinitesimal coordinate transformations $\left(\delta g_{\mu \nu}=\xi_{\mu ; \nu}+\xi_{\nu ; \mu}\right)$ vanishes because it is an invariant on the one hand but is equal, on the other, to

$$
\left(\delta I_{m} / \delta g\right) \delta g+\left(\delta I_{m} / \delta \psi\right) \delta \psi .
$$

The second term vanishes by virtue of the Euler-Lagrange equations; the first is just proportional to $\xi_{\nu} T_{; \mu}^{\mu \nu}$ for arbitrary $\xi_{\nu}$. See also S. Deser, Lett. Nuovo Cimento 1, 866 (1971).

${ }^{23}$ Integral representations of the Einstein equations do exist and could presumably be applied to the finite-mass equations as well. They are, however, rather different from the familiar Green's functions expressions. D. W. Sciama, P. C. Waylen, and R. C. Gilman, Phys. Rev. 187, 1762 (1969).

${ }^{24}$ It is possible to find a mass term such that the righthand side of Eq. (52a) is already $\tau_{\mu \nu}^{\text {tot }}$, i. e., where the nonlinear part of $f_{\mu \nu}$ is precisely $T_{\mu \nu}^{m}$. Unfortunately, $f^{L}$ then has a ghost structure $\left(f_{\mu \nu}^{L} \sim h_{\mu \nu}-\frac{1}{2} \eta_{\mu \nu} h_{\alpha}^{\alpha}\right)$ and is excluded on that ground. Details of the model are given in P. G. O. Freund, A. Maheswari, and E. Schonberg, Astrophys. J. 157, 857 (1969).

${ }^{25} \mathrm{~A}$ linear action for the $f$ field runs into contradictions; see C. Aragone and S. Deser, Nuovo Cimento 3A, 709 (1971). We also remark that one could investigate the stability of a finite-mass gravitational theory by the variational theory by the variational methods of that paper.

${ }^{26}$ C. J. Isham, Abdus Salam, and J. Strathdee, Phys. Rev. D 3,867 (1971). The mass term can also be expressed in terms of vierbeins rather than metric tensors. We have not investigated this variant which is given by J. Wess and B. Zumino [B. Zumino, in Lectures on Elementary Particles and Quantum Field Theory, edited by S. Deser et al . (MIT Press, Cambridge, Mass., (1971)] . ${ }^{27}$ P. W. Higgs, Phys. Letters 12, 132 (1964); Phys. Rev. 145,1156 (1966); G. S. Guralnik, C. R. Hagen, and T. W. B. Kibble, Phys. Rev. Letters 13, 585 (1964). 